\title{
Pembrolizumab Monotherapy in Relapsed Hepatocellular Carci- noma Post Living Donor Liver Transplantation and Sorafenib
}

\author{
Fadi Nasr ${ }^{1,2 *}$, Ahmad Al Ghoche ${ }^{1}$, Saada Diab ${ }^{1}$, Janah Maddah ${ }^{1}$, Layal Mansour ${ }^{1}$, Ali kassem ${ }^{1}$ and Abd \\ El Karim Gheith ${ }^{1}$
}

${ }^{1}$ Hematology-Oncology Department, Mount Lebanon Hospital, Beirut, Lebanon

${ }^{2}$ Department of Hematology-Oncology, Hotel-Dieu de France University Hospital, Faculty of Medicine, Saint Joseph University, Beirut, Lebanon

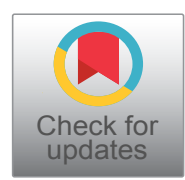

*Corresponding author: Fady Nasr, Head of Division, Hematology-Oncology Department, Hotel-Dieu de France University Hospital, Faculty of Medicine, Saint Joseph University, Mount Lebanon Hospital, P.O box: 470 Hazmieh, Beirut, Lebanon, Tel: +961-3-126606

\begin{abstract}
Background: Despite of the high incidence of hepatocellular carcinoma (HCC), treatment strategies stays restrained. New treatment aspects remain a necessity to improve overall survival of patients.

Case report: Here, we report for the first time a 2 years complete remission of hepatocellular carcinoma with pembrolizumab after failure of sorafenib and progression after liver transplantation.

Conclusion: Immunotherapy became a field of interest in $\mathrm{HCC}$ for researchers and clinicians in order to establish new immunotherapy modalities that improve response to treatment of HCC.
\end{abstract}

\section{Keywords}

Pembrolizumab, Sorafenib, Hepatocarcinoma, Transplant

\section{Abbreviations}

CT: Computerized Tomography; HCC: Hepatocellular Carcinoma; LT: Liver Transplantation

\section{Introduction}

HCC accounts for more than $90 \%$ of all cases of primary liver cancer [1]. It is the sixth most common solid cancer worldwide [2] and the third most common cause of cancer-related death in the world $[3,4]$. Treatment options for newly diagnosed liver carcinoma include liver transplantation, surgical resection, locoregional therapies $[5,6]$ and targeted therapies. The prognosis in the majority of advanced cases is usually dismal with only short-term survivals. Despite recent advances in the treatment of HCC, greater than $70 \%$ of HCC patients will not glean benefit from these interventions due to underlying liver dysfunction and/or presentation with advanced stage disease and, unfortunately, only $10-30 \%$ of patients with newly diagnosed HCC will be eligible for surgery. Treatment options for recurrence following liver transplantation (LT) for HCC are extremely limited [7]. Immune check point inhibitors are gaining role in an increasing number of cancers since there were first approved few years ago in metastatic melanoma [8], changing the therapeutic landscape and prognosis of many previously deemed incurable cancers. In this report, we present a rare case of transplanted hepatocellular carcinoma that was treated with immunotherapy post-progression after tyrosine kinase inhibitor and achieved long term survival.

\section{Case Presentation}

Our case was a 63-years-old Lebanese male patient, diabetic, with coronary artery disease status post angioplasty, with chronic hepatitis B on entecavir that was complicated by HCC. He underwent hepatectomy followed by liver transplantation in February 2012 and was started since then on immunosuppressive therapy with tacrolimus, mycophenolate mofetil and continued his entecavir for his chronic well controlled viral infec-

Citation: Nasr F, Al Ghoche A, Diab S, Maddah J, Mansour L, et al. (2018) Pembrolizumab Monotherapy in Relapsed Hepatocellular Carcinoma Post Living Donor Liver Transplantation and Sorafenib. Int J Oncol Res 1:009.

Accepted: November 10, 2018; Published: November 12, 2018

Copyright: (C) 2018 Nasr F, et al. This is an open-access article distributed under the terms of the Creative Commons Attribution License, which permits unrestricted use, distribution, and reproduction in any medium, provided the original author and source are credited. 
tion. In June 2012, patient was found to have anastomotic biliary stricture and a hepatico-jejunostomy was done with good outcome. He remained stable on regular follow up until May 2016 when he was admitted for new onset, recurrent episodes of productive cough. At this time, an injected computerized tomography (CT) scan of the chest was done and showed multiple lung nodules in the left upper and lower lobes. Biopsy of the lesions revealed metastatic moderately differentiated hepatocellular carcinoma with lympho vascular invasion compatible with the same initial liver biopsy. In front of these new findings, decision was to start sorafenib. CT abdomen and pelvis in June 2016 showed no evidence of disease in the transplanted liver but revealed adrenal metastasis with mesenteric soft tissue lymph nodes. No new biopsy was done at that time. In September 2016, while patient was still on sorafenib, he developed severe hand and foot syndrome deemed to be due to the tyrosine kinase inhibitor. So, the dose of sorafenib was reduced but no improvement in skin lesions was seen. In front of this treatment intolerance and confirmed progression of disease on CT scan, decision was to stop definitively sorafenib and to shift to immunotherapy with pembrolizumab at a dose of $200 \mathrm{mg}$ IV every 21 days. No other targeted therapy was used in order to avoid side effects observed with tyrosine kinase inhibitor. Positron emission tomography-computerized tomography (PET-CT) scan, in early January 2017, after 6 doses of pembrolizumab showed a complete remission with complete resolution of previously described lesions and no new lesions seen outside. After these positive results, we decided to continue pembrolizumab every 3 weeks with regular PET-CT and CT scans done every 3-4 months thereafter. Patient is currently still in complete remission with normal liver function and still maintained on dual immunosuppressive treatment with antiviral therapy.

\section{Discussion}

The immune checkpoint protein programmed death receptor 1(PD-1) is an inhibitory molecule expressed on the surface of multiple tissue types and keeps the $T$ cells from attacking tumor cells. Pembrolizumab is a humanized monoclonal immunoglobulin against PD-1 that blocks the signaling of the PD-1 receptor, thus reversing immunosuppression induced by cancer cells and restoring the immune system, allowing it to regain its original ability to attack cancer cells [9]. Recently, another immune check point inhibitor, nivolumab, was FDA approved for the treatment of advanced HCC cases treated with sorafenib [10]. In our case, we report a patient that have failed liver transplantation and palliative therapy with tyrosine kinase inhibitor and surprisingly achieved maintained complete remission till the current time (September 2018) with pembrolizumab. This is to our knowledge the first case reporting such long-term remission in transplanted relapsed hepatocellular carcinoma while still on immunosuppressive therapy with no clinical or laboratory evidence of graft rejection. In fact, it was not expected to have such a major response. But since the response was sustained after few months of therapy, we decided to continue treatment until progression. Regarding the contraindication of use previously described, the patient had no other therapeutic option due to tyrosine kinase toxicity added to progression, and after discussion with him, we elected to start a non-approved treatment in this setting in order to try to save his life since it is having major effects in other cancers. We do not have an explanation for such major sustained response. Tumor mutational burden study might be helpful in such setting. Also, tyrosine kinase use before immunotherapy could be a possible reason behind response in our case although not previously proven. The combination between immunosuppression and checkpoint inhibitors might have played a role in this setting. Previous case reported a complete remission in a liver donor recipient for hepatocellular carcinoma with the addition of pembrolizumab to the sorafenib [11] after progression on sorafenib monotherapy and other reports discussed the use of immunotherapy in relapsing or new onset cancers in transplanted patients with various outcomes between graft rejection, living with cancer or death $[12,13]$. In our case, the tyrosine kinase inhibitor was withheld due to toxicity and disease progression. In a recent report, 14 cases using immunotherapy (nivolumab or pembrolizumab) in the post-transplant setting, none of them had successful outcome with pembrolizumab in a hepatocellular carcinoma patient. The median survival time of patients following the diagnosis of unresectable disease is approximately 6-20 months, whereas long-term cure is achieved in fewer than 5\% of all patients [14] Five-year survival rate can reach $70 \%$ in patients with early HCC and preserved liver function $[15,16]$.

Another point to consider is the concomitant use of immunosuppressive therapy with immune check point inhibitors which mode of action is somewhat to boost immunity. Despite the increased need for effective cancer treatment options, check point inhibitors are considered as contraindicated in organ transplant recipients for fear of organ rejection [17]. The therapeutic success of such approach could be an important advance in the treatment of this category of patients where the choice between shifting to other tyrosine kinase inhibitors such as regorafenib $[16,18]$ or cabozantinib, starting ramucirumab [19] as efficacy has been recently shown in study, or shifting to end of life and hospice care. Many studies are ongoing to further assess the use of Pembrolizumab in advanced hepatocellular carcinoma cases $[20,21]$.

\section{Conclusion}

We report the first successful case of Pembrolizumab therapy in metastatic HCC after liver transplant on a background of post immunosuppression. 
It is well known that treatment options are limited for HCC which is one of the frequent cancer with high mortality rate. Such promising results add knowledge on immunotherapy use. PD-1 blockade maybe a new option of treatment for advanced HCC that failed transplant followed by sorafenib. Further studies are needed to support or decline such approach in this category of patients.

\section{Declarations}

\section{Acknowledgments}

We would like to thank the patient for his cooperation in this study.

\section{Funding}

There is no funding to report.

\section{Availability of data and materials}

The datasets used and analyzed during the present study are available from the corresponding author on reasonable request.

\section{Author's contributions}

$A G, S D$ collected data, designed and wrote the manuscript. JM, LM, AK, AG collected data. FN provided relevant scientific advice and revised the manuscript, analyzed and interpreted the patient data regarding clinical presentation, and treatment options. All listed authors contributed to critically revising the article and have approved the final version.

\section{Ethics approval and consent to participate}

Research involving oncology analyses was approved by the Institutional Review Board at the Mount Lebanon hospital and written informed consent obtained from the patient described herein, in accordance with applicable local and international legal and ethical standards, including the Declaration of Helsinki of the World Medical Association.

\section{Consent for publication}

The patient presented in this case report has explicitly consented to publishing his medical history in the present form. Respective written informed consent was obtained by the authors.

\section{Competing interests}

The authors declare that they have no competing interests.

\section{References}

1. https://www.elsevier.com/books/robbins-and-cotran-pathologic-basis-of-disease/kumar/978-1-4557-2613-4

2. https://www.wcrf.org/dietandcancer/cancer-trends/liver-cancer-statistics

3. Ferlay J, Soerjomataram I, Ervik M, Dikshit R, Eser S, et al. (2012) GLOBOCAN 2012: Estimated cancer incidence, mortality and prevalence worldwide in 2012 v1.0.

4. https://seer.cancer.gov/statfacts/

5. Jemal A, Siegel R, Xu J, Ward E (2010) Cancer statistics, 2010. CA Cancer J Clin 60: 277-300.

6. Bosch FX, Ribes J, Díaz M, Cléries R (2004) Primary liver cancer: Worldwide incidence and trends. Gastroenterology 127: S5-S16.

7. Peter J Kneuertz, David P Cosgrove, Andrew M Cameron, Ihab R Kamel, Jean Francois H Geschwind, et al. (2012) Multidisciplinary management of recurrent hepatocellular carcinoma following liver transplantation. J Gastrointest Surg 16: 874-881.

8. https://news.bms.com/press-release/rd-news/fda-approves-yervoy-ipilimumab-treatment-patients-newly-diagnosed-or-previousl

9. Kudo M (2016) Immune checkpoint blockade in hepatocellular carcinoma: 2017 Update. Liver Cancer 6: 1-12.

10. https://www.fda.gov/drugs/informationondrugs/approveddrugs/ucm577166.htm

11. Chen SC, Chao Y, Yang MH (2017) Complete Response to the Combination of Pembrolizumab and Sorafenib for Metastatic Hepatocellular Carcinoma: A Case Report. Am J Gastroenterol 112: 659-660.

12. Biondani P, De Martin E, Samuel D (2018) Safety of an anti-PD-1 immune checkpoint inhibitor in a liver transplant recipient. Ann Oncol 29: 286-287.

13. Varkaris A, Lewis DW, Nugent FW (2017) Preserved liver transplant after PD-1 pathway inhibitor for hepatocellular carcinoma. Am J Gastroenterol 112: 1895-1896.

14. Mor E, Tur Kaspa R, Sheiner P, Schwartz M (1998) Treatment of hepatocellular carcinoma associated with cirrhosis in the era of liver transplantation. Ann Intern Med 129: 643653.

15. Llovet JM, Fuster J, Bruix J (1999) Intention-to-treat analysis of surgical treatment for early hepatocellular carcinoma: Resection versus transplantation. Hepatology 30: 14341440.

16. Poon RT, Fan ST, Lo CM, Liu CL, Wong J (2002) Longterm survival and pattern of recurrence after resection of small hepatocellular carcinoma in patients with preserved liver function: Implications for a strategy of salvage transplantation. Ann Surg 235: 373-382.

17. Munker S, De Toni EN (2018) Use of checkpoint inhibitors in liver transplant recipients. United Eur Gastroenterol J 6: 970-973.

18. Bruix J, Qin S, Merle P, Granito A, Huang YH, et al. (2017) Regorafenib for patients with hepatocellular carcinoma who progressed on sorafenib treatment (RESORCE): A randomised, double-blind, placebo-controlled, phase 3 trial. The Lancet 389: 56-66.

19. https://www.prnewswire.com/news-releases/lilly-announces-cyramza-ramucirumab-phase-3-reach-2-study-in-second-line-hepatocellular-carcinoma-patients-met-overall-survival-endpoint-300624113.html

20. https://clinicaltrials.gov/ct2/show/NCT02702414

21. Zhu AX, Finn RS, Edeline J, Cattan S, Ogasawara S, et al. (2018) Pembrolizumab in patients with advanced hepatocellular carcinoma previously treated with sorafenib (KEYNOTE-224): A non-randomised, open-label phase 2 trial. Lancet Oncol 19: 940-952. 